\title{
Preterm cerebellum at term age: ultrasound measurements are not different from infants born at term
}

\author{
André M. Graça' ${ }^{1}$ Ana Filipa Geraldo², Katia Cardoso' and Frances M. Cowan ${ }^{3}$
}

BACKGROUND: Reduced supratentorial brain growth has been shown in preterm-born infants at term-equivalent age (TEA), but cerebellar growth may be preserved in the absence of supratentorial injury. Our study aims to compare cerebellar size assessed using cerebral ultrasound (CUS) at TEA between preterm infants and term-born controls.

METHODS: Cerebellar dimensions (including transverse cerebellar diameter (TCD), cerebellar vermis height, anteroposterior vermis diameter (APVD), and cerebellar vermis area (CVA)) were measured using Image Arena software (TomTec Imaging Systems, Unterschleissheim, Germany) in 71 infants <32-wk gestation without significant scan abnormality at TEA and in 58 term-born control infants. Intra- and interobserver agreement were evaluated.

RESULTS: In comparison with controls, preterms at TEA had smaller TCDs (4.9 vs. $5.2 \mathrm{~cm} ; P<0.001)$ but larger CVAs (4.7 vs. $\left.4.3 \mathrm{~cm}^{2} ; P<0.005\right)$ and APVDs (2.4 vs. $2.2 \mathrm{~cm} ; P<0.001$ ); however, these differences were no longer seen after accounting for head shape. In <28-wk gestational age infants, CVA was statistically similar to controls, as were for small-for-gestationalage infants.

CONCLUSION: Our data support neonatal sparing of preterm cerebellar growth that is measureable using CUS, and this includes the most immature and small-for-gestational-age infants. We suggest cUS can be used to assess cerebellar size at TEA, with measures of both width and height being taken into account, and thus may be a useful tool for detecting infants with poorer cerebellar growth who are at increased risk of disability.

A n extrauterine environment is adverse for brain development of preterm infants (1-3). Survival of very preterm infants and survival without neurodevelopmental disabilities have improved (4) with better outcomes for very immature infants born in the last decade as compared with those born in the 1990s (5). Neurodevelopmental disabilities of very preterm infants can be partly predicted by the presence of major lesions, either supratentorial or cerebellar, which can be detected using cerebral ultrasound (cUS) (6) or magnetic resonance imaging (MRI) $(7,8)$. Even in the absence of major lesions, reduced supratentorial brain growth in preterms at term-equivalent age (TEA) has been shown using MRI $(9,10)$ and cUS $(11,12)$. The predictive value of such a reduced growth for neurodevelopmental outcomes is still not clearly evaluated $(9,12,13)$.

In contrast to reduced supratentorial brain growth, cerebellar growth in preterm infants has been shown in some studies to be preserved at TEA in the absence of major supratentorial injury $(14,15)$, although other studies have found different results $(16,17)$. Studies, performed in childhood, in survivors of preterm birth, suggest smaller cerebellar sizes than that in term-born controls, which are related to adverse neurodevelopmental outcomes $(15,18-20)$. It is not yet known whether an insult sustained during the neonatal period in preterms may lead to arrested cerebellar development later in childhood, even in infants with normal cerebellar size at TEA $(19,20)$.

Studies on cerebellar size and growth have been largely performed using MRI $(14,21)$. The aims of this study are to evaluate cerebellar size at TEA using cUS in very preterm infants without major brain lesions and to compare it with cerebellar size in a control group of healthy term infants. In addition, we wish to evaluate cerebellar size at TEA in preterms of different birth gestational ages (GA) and in small-for-gestational-age (SGA) preterm infants. We also aim to determine if neonatal risk factors were associated with cerebellar size at TEA.

\section{RESULTS}

A total of 126 infants were assessed: 70 preterms at TEA and 56 term infants during their first postnatal week (Table 1). No infant in the control group was invasively ventilated, although nine had nasal continuous positive airway pressure (median 2d) for transient tachypnoea; $39 \%$ were given antibiotics for C-reactive protein $>2 \mathrm{mg} / \mathrm{dl}$ plus risk factors for early infection, but none was symptomatic or had culture-proven sepsis. By contrast, $44 \%$ of the preterm infants were invasively ventilated; $54 \%$ had at least one episode of clinical sepsis, half of whom had a positive blood culture. No significant differences between the groups were found for gender or being SGA at birth.

Cerebral ultrasound was performed at a mean age of $12 \mathrm{wk}$ in preterms and $5 \mathrm{~d}$ in controls. No term infant was scanned before $48 \mathrm{~h}$. Postmenstrual age (PMA) at scan was similar between the groups. Weight and length on scan date were

'Departmento de Pediatria, Serviço de Neonatologia, Hospital de Santa Maria/Centro Académico de Medicina de Lisboa, Lisbon, Portugal; ${ }^{2}$ Serviço de Imagiologia Neurológica, Hospital de Santa Maria/Centro Académico de Medicina de Lisboa, Lisbon, Portugal; ${ }^{3}$ Department of Paediatrics, Imperial College/Hammersmith Hospital, London, UK. Correspondence: André M. Graça (graca.am@gmail.com) 
smaller in preterms, whereas mean occipitofrontal circumference was $9 \mathrm{~mm}$ larger in the preterm group (Table 1 ).

Transverse cerebellar diameter (TCD) measurements made via both the anterior fontanelle (AF) and mastoid fontanelle (MF) were available in 19 preterm and 16 term infants. The mean difference between these approaches was $-0.5 \mathrm{~mm}$ (95\% confidence interval for the mean $=-0.9$ to $-0.1 \mathrm{~mm}$ ), and the intraclass correlation coefficient (ICC) was 0.936 , confirming no significant difference between the TCD measured via the $\mathrm{AF}$ or the MF.

Table 1. Patient characteristics of preterm cohort and term-born control infants

\begin{tabular}{|c|c|c|c|}
\hline $\begin{array}{l}\text { Characteristics } \\
\text { of patients }\end{array}$ & Preterm & Term & $P$ \\
\hline Number & 70 & 56 & \\
\hline Birth weight $(g)^{a}$ & $1,073(510-1,515)$ & $3,191(2,385-4,445)$ & $<0.001$ \\
\hline $\begin{array}{l}\text { Gestational age } \\
(w k)^{\mathrm{a}}\end{array}$ & $28.6(24-32)$ & $39.2(37-42)$ & $<0.001$ \\
\hline $\begin{array}{l}\text { Small for } \\
\text { gestational age }\end{array}$ & $19 \%$ & $13 \%$ & NS \\
\hline Male sex ${ }^{b}$ & $54 \%$ & $61 \%$ & NS \\
\hline $\begin{array}{l}\text { Caesarean } \\
\text { section }^{\mathrm{b}}\end{array}$ & $74 \%$ & $53 \%$ & $<0.02$ \\
\hline $\begin{array}{l}\text { Apgar score at } \\
1 \min ^{c}\end{array}$ & $8(1-10)$ & $9(3-10)$ & $<0.001$ \\
\hline $\begin{array}{l}\text { Apgar score at } \\
5 \min ^{c}\end{array}$ & $10(3-10)$ & $10(8-10)$ & $<0.01$ \\
\hline CRIB score $^{c}$ & $1(0-11)$ & $0(0-4)$ & $<0.001$ \\
\hline $\begin{array}{l}\text { Invasive } \\
\text { ventilation }^{\mathrm{b}}\end{array}$ & $46 \%$ & $0 \%$ & $<0.001$ \\
\hline $\begin{array}{l}\text { Chronic lung } \\
\text { disease }^{\mathrm{b}}\end{array}$ & $17 \%$ & $0 \%$ & $<0.002$ \\
\hline $\begin{array}{l}\text { Hypotension } \\
\text { with inotropes }^{d}\end{array}$ & $7 \%$ & $0 \%$ & NS \\
\hline $\begin{array}{l}\text { Patent ductus } \\
\text { arteriosus }^{\mathrm{b}}\end{array}$ & $26 \%$ & $0 \%$ & $<0.001$ \\
\hline $\begin{array}{l}\text { Necrotizing } \\
\text { enterocolitis }^{d}\end{array}$ & $6 \%$ & $0 \%$ & NS \\
\hline $\begin{array}{l}\text { Sepsis (culture } \\
\text { proven) }^{\mathrm{b}}\end{array}$ & $27 \%$ & $0 \%$ & $<0.001$ \\
\hline Age at scan $(d)^{a}$ & $84(49-124)$ & $5(2-9)$ & $<0.001$ \\
\hline $\begin{array}{l}\text { PMA at scan } \\
(w k)^{\mathrm{a}}\end{array}$ & $40.2(37-44)$ & $39.9(38-43)$ & NS \\
\hline $\begin{array}{l}\text { Weight at scan } \\
(\mathrm{g})^{\mathrm{e}}\end{array}$ & $2,821(1,730-4,000)$ & $3,176(2,235-4,570)$ & $<0.001$ \\
\hline $\begin{array}{l}\text { Length at scan } \\
(\mathrm{cm})^{\mathrm{e}}\end{array}$ & $46.7(40-53)$ & $49.2(45-52)$ & $<0.001$ \\
\hline $\begin{array}{l}\text { Occipital-frontal } \\
\text { circumference at } \\
\text { scan }(\mathrm{cm})^{\mathrm{e}}\end{array}$ & $35.4(30-40)$ & $34.5(32-38)$ & $<0.01$ \\
\hline
\end{tabular}

CRIB, clinical risk index for babies; NS, not significant; PMA, postmenstrual age.

aPresented as mean (range), statistical analysis using Mann-Whitney test. ${ }^{\text {bP Presented }}$ as percentage, statistical analysis using Pearson $x^{2}$ test. 'Presented as median (range), statistical analysis using Mann-Whitney test. dPresented as percentage, statistical

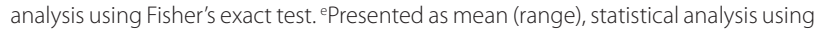
independent sample $t$ test.
In comparison with term infants, preterms at TEA, including those who were SGA, had smaller TCD $(4.9$ vs. $5.2 \mathrm{~cm} ; P<$ $0.001)$ but larger cerebellar vermis area (CVA) $\left(4.7\right.$ vs. $4.3 \mathrm{~cm}^{2}$; $P<0.005)$, larger anteroposterior vermis diameter $(2.4$ vs. $2.2 \mathrm{~cm} ; P<0.001)$, and larger anteroposterior pons diameter (1.8 vs. $1.7 \mathrm{~cm} ; P<0.001)$. Cerebellar vermis height, fourth ventricle area, fourth ventricle depth, and cisterna magna height $(\mathrm{CMH})$ were not statistically different between the two groups (Table 2).

Despite a small, nonsignificant difference between groups for PMA, we tested for an eventual effect of that difference on our results using ANCOVA analysis. Observed differences in the mean values for TCD and CVA for preterms at TEA and term control infants did not change after correcting those values for PMA.

We have previously found significant differences in head shape between term control and preterms at TEA (11), so we tested the hypothesis that our measurements might be influenced by head shape by applying ANCOVA-corrected models for the two main variables:

1. After correcting TCD for biparietal diameter, we found no significant difference between groups $(5.0 \mathrm{vs} .5 .1 \mathrm{~cm}$; not significant);

2. After correcting CVA for cranial height and anteroposterior cranial diameter, we found no significant difference between groups ( 4.5 vs. $4.6 \mathrm{~cm}^{2}$; not significant).

Table 2. Comparison of cerebellar dimensions between the complete preterm cohort at term-equivalent age and term-born control infants

\begin{tabular}{lccc}
\hline & Preterm & Term & $P$ \\
\hline $\begin{array}{l}\text { Gestational age at } \\
\text { birth }(\text { wk) }\end{array}$ & $28.6(24-32)$ & $39.2(37-42)$ & $<0.001$ \\
$\begin{array}{l}\text { Postmenstrual age at } \\
\text { scan }(\text { wk) }\end{array}$ & $40.3(37-44)$ & $39.9(38-43)$ & NS \\
$\begin{array}{l}\text { Transverse cerebellar } \\
\text { diameter }(\mathrm{cm})^{\mathrm{b}}\end{array}$ & $4.9(4.0-5.2)$ & $5.2(4.5-6.0)$ & $<0.001$ \\
$\begin{array}{l}\text { Sagittal cerebellar } \\
\text { vermis area }\left(\mathrm{cm}^{2}\right)^{\mathrm{a}}\end{array}$ & $4.7(3.2-6.4)$ & $4.3(3.2-5.7)$ & $<0.005$ \\
$\begin{array}{l}\text { Anteroposterior vermis } \\
\text { diameter }(\mathrm{cm})^{\mathrm{a}}\end{array}$ & $2.4(1.8-2.9)$ & $2.2(1.6-2.6)$ & $<0.001$ \\
$\begin{array}{l}\text { Cerebellar vermis } \\
\text { height }(\mathrm{cm})^{\mathrm{a}}\end{array}$ & $2.4(1.9-2.9)$ & $2.3(2.0-2.8)$ & $\mathrm{NS}$ \\
$\begin{array}{l}\text { Anteroposterior pons } \\
\text { diameter }(\mathrm{cm})^{\mathrm{a}}\end{array}$ & $1.8(1.5-2.1)$ & $1.7(1.5-1.9)$ & $<0.001$ \\
$\begin{array}{l}\text { Fourth ventricle } \\
\text { sagittal area }(\mathrm{cm})^{\mathrm{b}}\end{array}$ & $0.1(0.0-0.2)$ & $0.1(0.0-0.3)$ & $\mathrm{NS}$ \\
$\begin{array}{l}\text { Fourth ventricle } \\
\text { sagittal depth }(\mathrm{cm})^{\mathrm{b}}\end{array}$ & $0.4(0.2-0.8)$ & $0.4(0.2-0.7)$ & $\mathrm{NS}$ \\
$\begin{array}{l}\text { Cisterna magna sagittal } \\
\text { height }(\mathrm{cm})^{\mathrm{a}}\end{array}$ & $0.6(0.3-1.0)$ & $0.6(0.4-0.8)$ & $\mathrm{NS}$ \\
\hline
\end{tabular}

NS, not significant.

apresented as mean (range), statistical analysis using $t$ test for independent samples. bPresented as mean (range), statistical analysis using Mann-Whitney test. 
Although there were no significant differences in PMA between the two groups, there was a range of PMA, and we were therefore able to examine the effect of PMA on our measurements. We found, as expected, an increase in cerebellar dimensions with increasing PMA and observed similar rates of increase for TCD $(\sim 0.6 \mathrm{~mm} / \mathrm{wk})$ and CVA $\left(\sim 8 \mathrm{~mm}^{2} / \mathrm{wk}\right)$ for preterms at TEA and term controls over this time period (Figure 1).

Intra- and interobserver agreement assessed using ICC was very good or good for all the measurements except for the interobserver agreement for CMH (Table 3).

\section{Clinical Risk Factors and Main Cerebellar Dimensions in Preterm Infants}

Subgroup analysis of the more immature infants born before 28-wk GA in comparison with the control group (Table 4) revealed no significant difference for any of the variables except for TCD, which was wider in term-born infants ( 4.8 vs. $5.2 \mathrm{~cm}$; $P<0.001)$ before correcting for head shape but not after applying the correction ( 5.0 vs. $5.1 \mathrm{~cm}$; not significant). On comparing cerebellar dimensions between preterm infants born before and after 28-wk GA, no difference was found except for a barely significant difference in CVA (Table 4).

The effect of being SGA was assessed (Table 5) by comparing SGA preterms $(n=13)$ with appropriate-for-gestationalage preterms $(n=57)$ and also by comparing appropriate-forgestational-age preterms with appropriate-for-gestational-age term infants $(n=49)$. The two preterm groups were of similar GA at birth. No statistical difference was found between SGA and appropriate-for-gestational-age preterm infants, except for a small though statistically significant differences in cerebellar vermis height $(2.2$ vs. $2.4 \mathrm{~cm} ; P<0.02)$ and anteroposterior pons diameter $(1.7$ vs. $1.8 \mathrm{~cm} ; P<0.03)$. After excluding SGA

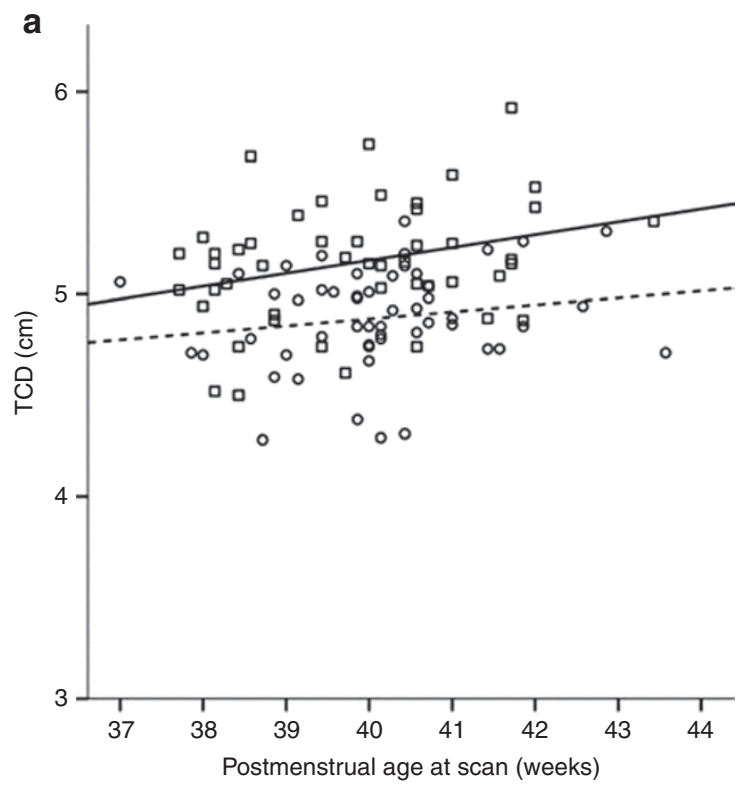

infants from both preterm and control groups, the findings were similar to the original whole-cohort comparison (Tables 2 and 5).

On bivariate analysis of risk factors for lower TCD, we found significant differences for the five preterm infants with hypotension requiring vasoactive therapy $(4.1 \mathrm{vs.} 4.9 \mathrm{~cm} ; P<0.001)$ and for the four preterm infants who had necrotizing enterocolitis (NEC; 4.4 vs. $4.9 \mathrm{~cm} ; P<0.01$ ) but found no significant differences for those requiring resuscitation at birth, invasive ventilation, treatment for patent ductus arteriosus, having at least one episode of culture-proven sepsis, or developing chronic lung disease (CLD). Weak or nonsignificant correlations were found for GA, birth weight, clinical risk index for babies score, or ventilation days with TCD at TEA. On linear regression analysis of risk factors for lower TCD, number of ventilation days $(B=0.008 ; P<0.05)$, NEC $(B=-0.302 ; P$ $<0.05)$, hypotension $(B=-0.701 ; P<0.001)$, and GA $(B=$ $0.056 ; P<0.02)$ remained significant as explanatory variables (adjusted $R^{2}=0.478 ; P<0.001$ ).

On bivariate analysis of risk factors for lower CVA, a significant difference was found only for those who were invasively ventilated ( 4.5 vs. $\left.4.8 \mathrm{~cm}^{2} ; P<0.02\right)$. None of the other clinical variables studied was significant for CVA dimension at TEA. On linear regression analysis, only birth weight $(B=0.001 ; P<$ $0.01)$ remained significant as an explanatory variable for CVA (adjusted $R^{2}=0.113 ; P<0.01$ ).

\section{DISCUSSION}

Our cUS data suggest that there is a relative sparing of cerebellar hemisphere and vermis growth up to TEA in very preterm infants without major brain lesions. These findings are in line with MR studies that show conserved cerebellar volumes of preterm infants at TEA in the absence of major supratentorial

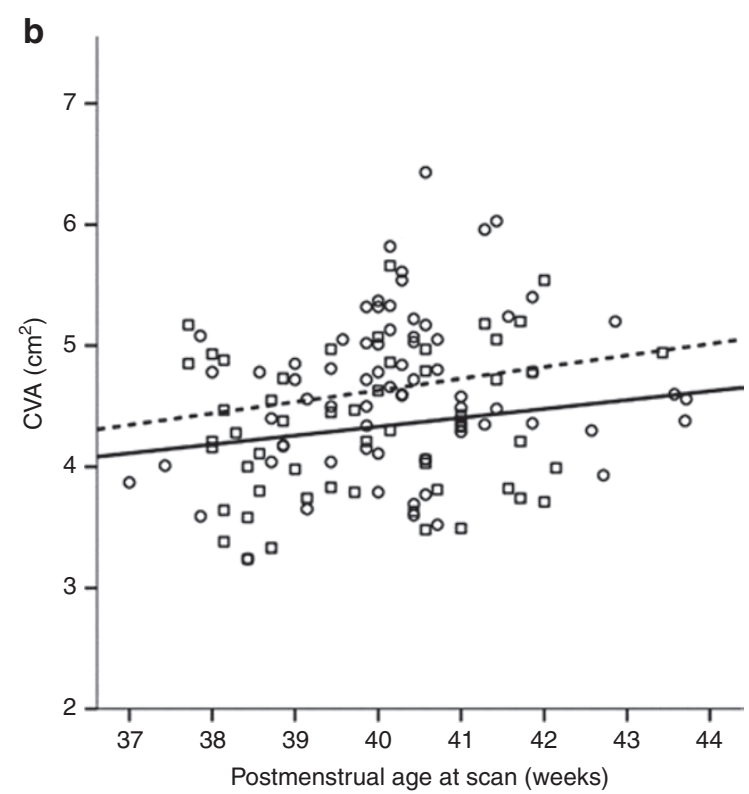

Figure 1. Graphs showing the relationship between cerebellar dimensions and postmenstrual age at scan: (a) TCD ( $\left(\mathrm{cm}^{2}\right)$ and $(\mathbf{b})$ CVA $\left(\mathrm{cm}^{2}\right)$. Preterm infants at TEA are represented by circles and term-born control infants are represented by squares. Mean fit lines are represented by dotted lines for preterm infants and by continuous lines for term-born controls. CVA, cerebellar vermis area; TCD, transverse cerebellar diameter; TEA, term-equivalent age. 
injury $(14,15)$. We found that this sparing effect was maintained even when analyzing data from the more immature and SGA preterm infants. As we only evaluated preterm infants without major cerebral lesions, we are unable to confirm the effect of supratentorial lesions on cerebellar dimensions at TEA previously described using MRI $(17,21)$. Since most reports showing reduced cerebellar size at TEA refer to associations of decreased growth when other brain lesions are also present, we speculate that the cerebellum itself is able to grow relatively normally in the absence of any negative trophic transsynaptic effects of disrupted connectivity between the cerebellum and supratentorial structures, which has been described both for unilateral and diffuse neonatal brain disorders (17).

We found that, when compared with term controls, preterm infants at TEA have a larger CVA but a narrower TCD. These findings may be partly explained by the preterm head shape at TEA, which is significantly longer and narrower in preterm infants when compared with term-born infants (11). However, when we corrected the TCD and CVA measurements for intracranial dimensions, we found that the differences between TCD and CVA in the two groups were no

Table 3. Intra- and interobserver reliability evaluation using intraclass correlation coefficient

\begin{tabular}{lcc}
\hline Cerebellar dimension & Intraobserver & Interobserver \\
\hline Transverse cerebellar diameter & $0.854^{+}$ & $0.593^{+}$ \\
Sagittal vermis area & $0.953^{+}$ & $0.809^{+}$ \\
Anteroposterior vermis diameter & $0.612^{+}$ & $0.812^{+}$ \\
Cerebellar vermis height & $0.917^{\dagger}$ & $0.706^{+}$ \\
Anteroposterior pons diameter & $0.862^{+}$ & $0.860^{+}$ \\
Fourth ventricle sagittal area & $0.643^{+}$ & $0.826^{+}$ \\
Fourth ventricle sagittal depth & $0.779^{+}$ & $0.840^{+}$ \\
Cisterna magna sagittal height & $0.646^{\dagger}$ & $0.256^{\ddagger}$ \\
\hline
\end{tabular}

${ }^{\dagger} P<0.001 ; \ddagger P<0.05$. longer apparent suggesting a similar global cerebellar size between preterms at TEA and term controls. This preserved cerebellar growth in preterms is further supported by the findings that the rate of increase in cerebellar size around TEA as measured from the TCD and CVA was similar for preterms and term infants.

Clinical risk factors for a smaller cerebellum at TEA other than major cerebral lesions have been described $(21,22)$ : notably postnatal steroid exposure, requirement for early intubation, hypotension, and patent ductus arteriosus. In our low-risk cohort, there were very small numbers of infants with those risk factors. Nevertheless, hypotension requiring vasoactive drugs was a significant risk factor for lower TCD and had a nonsignificant trend toward a smaller CVA. Patent ductus arteriosus, CLD, and postnatal steroid exposure were not associated with significantly different cerebellar dimensions at TEA. A selective effect of postnatal steroid exposure on cerebellar size at TEA, observed more often when dexamethasone is used, was recently reported as the greatest risk factor after intraventricular hemorrhage for a smaller cerebellar volume on MRI at TEA (22). In our study, only three infants were treated with postnatal steroids for severe CLD, and hydrocortisone was used in all cases. NEC was also significant as a risk factor for lower TCD in bivariate and a linear regression model. This reduction in cerebellar size of infants developing NEC is in line with previously described data by our group on this same cohort (11) showing a very significant difference between estimated brain size at term of infants who had NEC.

Despite having found a statistically significant difference of $1 \mathrm{~mm}$ for anteroposterior pons diameter favoring the preterm infants at TEA, this difference may be due to the cranial configuration of preterms at term, rather than indicating any real difference in pons size. The fact that we did not measure the pons in any other axis does not allow further speculation concerning the real pons size.

Table 4. Comparison of cerebellar dimension between the most immature infants of the preterm cohort, the remaining preterm infants, and the term infants

\begin{tabular}{|c|c|c|c|c|c|}
\hline & $<28 w k$ & $28-32 w k$ & $P^{a}$ & Term & $P^{\mathrm{b}}$ \\
\hline Number of babies & 24 & 46 & & 56 & \\
\hline Gestational age at birth (wk) & $26.6(24-28)$ & $29.7(28-32)$ & $<0.001$ & $39.2(37-42)$ & $<0.001$ \\
\hline Transverse cerebellar diameter $(\mathrm{cm})$ & $4.8(4.0-5.6)$ & $4.9(4.1-5.4)$ & NS & $5.2(4.5-6.0)$ & $<0.001$ \\
\hline Sagittal vermis area $\left(\mathrm{cm}^{2}\right)$ & $4.4(3.2-5.5)$ & $4.8(3.6-6.4)$ & $<0.05$ & $4.3(3.2-5.7)$ & NS \\
\hline Cerebellar vermis height $(\mathrm{cm})$ & $2.3(1.9-2.6)$ & $2.4(2.0-2.9)$ & NS & $2.3(2.0-2.8)$ & NS \\
\hline Anteroposterior pons diameter $(\mathrm{cm})$ & $1.8(1.5-2.0)$ & $1.8(1.6-2.1)$ & NS & $1.7(1.5-1.9)$ & NS \\
\hline Fourth ventricle sagittal area $\left(\mathrm{cm}^{2}\right)$ & $0.1(0.0-0.2)$ & $0.1(0.0-0.2)$ & NS & $0.1(0.0-0.3)$ & NS \\
\hline Fourth ventricle sagittal depth $(\mathrm{cm})$ & $0.4(0.2-0.8)$ & $0.4(0.2-0.8)$ & NS & $0.4(0.2-0.7)$ & NS \\
\hline Cisterna magna sagittal height $(\mathrm{cm})$ & $0.6(0.4-0.8)$ & $0.7(0.3-1.0)$ & NS & $0.6(0.4-0.8)$ & NS \\
\hline
\end{tabular}

All data presented as mean (range), statistical analysis using $t$ test for independent samples or Mann-Whitney test as appropriate.

NS, not significant.

a $P$ value for comparison of preterm infants $<28$ wk and between 28 and $32 \mathrm{wk}$. ${ }^{\mathrm{b}} \mathrm{P}$ value for comparison of preterm infants $<28 \mathrm{wk}$ and term-born controls. 
Table 5. Evaluation of the effect of being SGA on cerebellar size

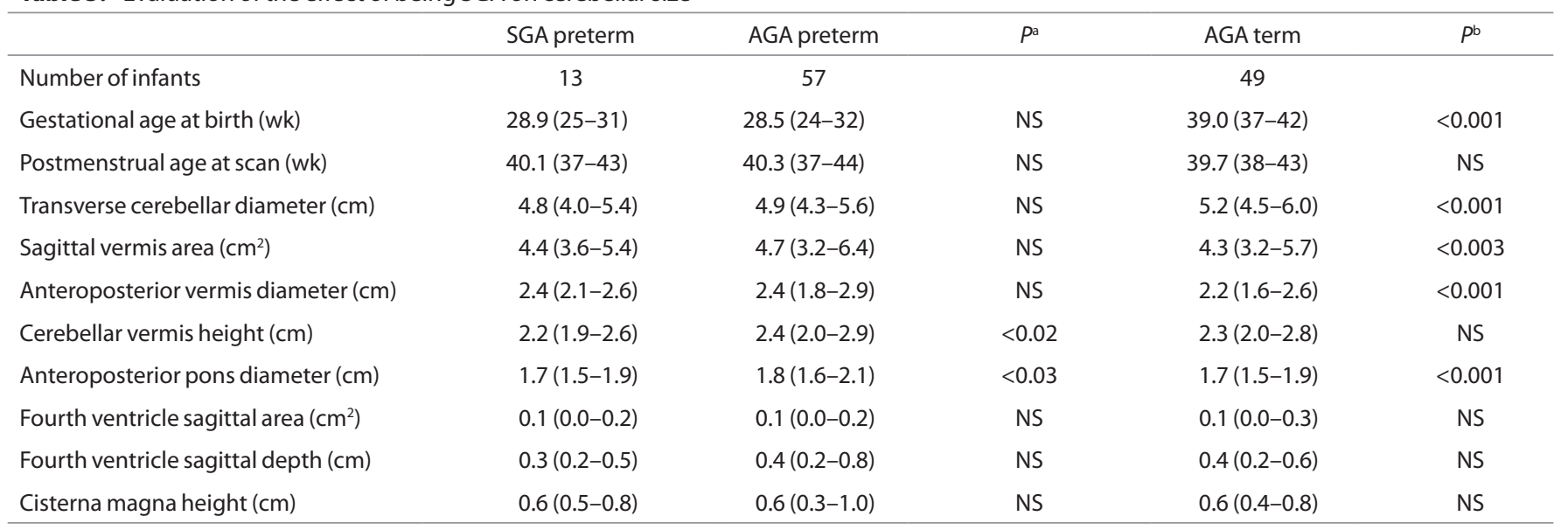

All data presented as mean (range); statistical analysis using $t$ test for independent samples or Mann-Whitney test as appropriate.

AGA, appropriate for gestational age; NS, not significant; SGA, small for gestational age.

a $P$ value for comparisons between SGA- and AGA-preterm infants. ${ }^{b} P$ value for comparisons between AGA preterm and AGA term infants.

We also did not find any significant difference for CM size between the groups. This finding differs from the general extracerebral space size described previously by our group in this same sample (11), which was much larger in the preterms at TEA. We speculate that this finding supports our conclusion that cerebellar size is relatively preserved in preterms at TEA.

Intra- and interobserver reliability analysis is very important for future application of a concept that may be used clinically. We found good or very good reliability for the key measurements made for this article (TCD and CVA). Nevertheless, we only found a fair reliability for interobserver reliability of $\mathrm{CMH}$. This may be explained by the absence of clear reference points both on the posterior lip of the foramen magnum and the lower limit of the vermis, and care needs to be taken in avoiding echoes that come from the cerebellar tonsils rather than the vermis.

Our comparison of TCD measured via both the anterior and MF showed the same excellent concordance that we had found in preterm infants (23). Occasionally, it can be difficult to make good measurements of the TCD by one or other acoustic window; these data confirm that once a good image is obtained, the measurements are not different, and either approach can be used.

Follow-up of these infants is ongoing to assess whether there is any relationship between cerebellar (as well as other measures we have made) dimensions and neurodevelopmental outcomes. However, given the similar cerebellar values found for the preterm infants included in this study and term controls, we would not expect very relevant prognostic value of ultrasound measurements for outcome. Nevertheless, our results aid in establishing normal values for these measurements that are helpful in defining cerebellar hypoplasia resulting from localized cerebellar pathology or the atrophic effect of supratentorial lesions. Infants who have a smaller cerebellum at TEA may be at increased risk of neurodevelopmental problems $(24,25)$ and may benefit both from a MRI scan at TEA for further definition of pathology, aiding in predicting outcome, and closer neurodevelopmental follow-up.

\section{METHODS}

We assessed prospectively cerebellar dimensions in a cohort of infants born at $<32$-wk GA and scanned at TEA and in a group of healthy, term-born control infants scanned during the first postnatal week.

The study was performed at the Hospital de Santa Maria in Lisbon, a tertiary neonatal intensive care unit and main referral center in South Portugal for neonatal neurology, neurosurgery, and metabolic disorders. Written informed parental consent was obtained for each case and control infant; the study was approved by the Medical Ethics Committee of the Hospital de Santa Maria.

Preterm infants with neurological problems or any cerebellar or supratentorial scan abnormality up to TEA, (except isolated germinal matrix hemorrhage or transient periventricular flares that did not evolve to cystic periventricular leukomalacia and did not persist to TEA) were excluded from the study. SGA infants (birth weight $<10$ th centile on the updated Babson and Benda's charts) (26) were not excluded from the initial cohort but were also analyzed separately.

The term control infants were either term-born infants recruited from the postnatal ward (one-third) or infants on the low-dependency care neonatal unit (two-third); some had jaundice, were being given antibiotics for suspected but unproven sepsis, or mild and transient respiratory distress. Median Apgar scores were 9 at $1 \mathrm{~min}$ and 10 at $5 \mathrm{~min}$. The infants neither had abnormality detected using cUS nor had neonatal neurological problems. They were born during the study period and remained in the hospital for at least $72 \mathrm{~h}$.

Clinical data were collected in relation particularly to antenatal growth, condition at birth and postnatal course with respect to clinical risk index for babies scores (27), length of ventilation, nasal continuous positive airway pressure, CLD (defined as oxygen supplementation after 36-wk PMA), postnatal steroid use for CLD, hypotension requiring the use of inotropes, blood culture-proven sepsis, NEC, days on parenteral nutrition, patent ductus arteriosus, and retinopathy of prematurity.

All images were acquired by one author (A.M.G.) using a Siemens Acuson Sequoia scanner (Siemens, Erlangen, Germany) with a 10v4 transducer set to $8.5 \mathrm{MHz}$ and digitally stored in the Digital Imaging and Communications in Medicine format. cUS scans of preterm infants were performed as part of our standard neonatal intensive care unit scanning protocol. Scanning of term-born control infants was performed specifically for this study, but the scanning was avoided during the first $72 \mathrm{~h}$ after birth to allow time to minimize the headmoulding effects on the measurements. 

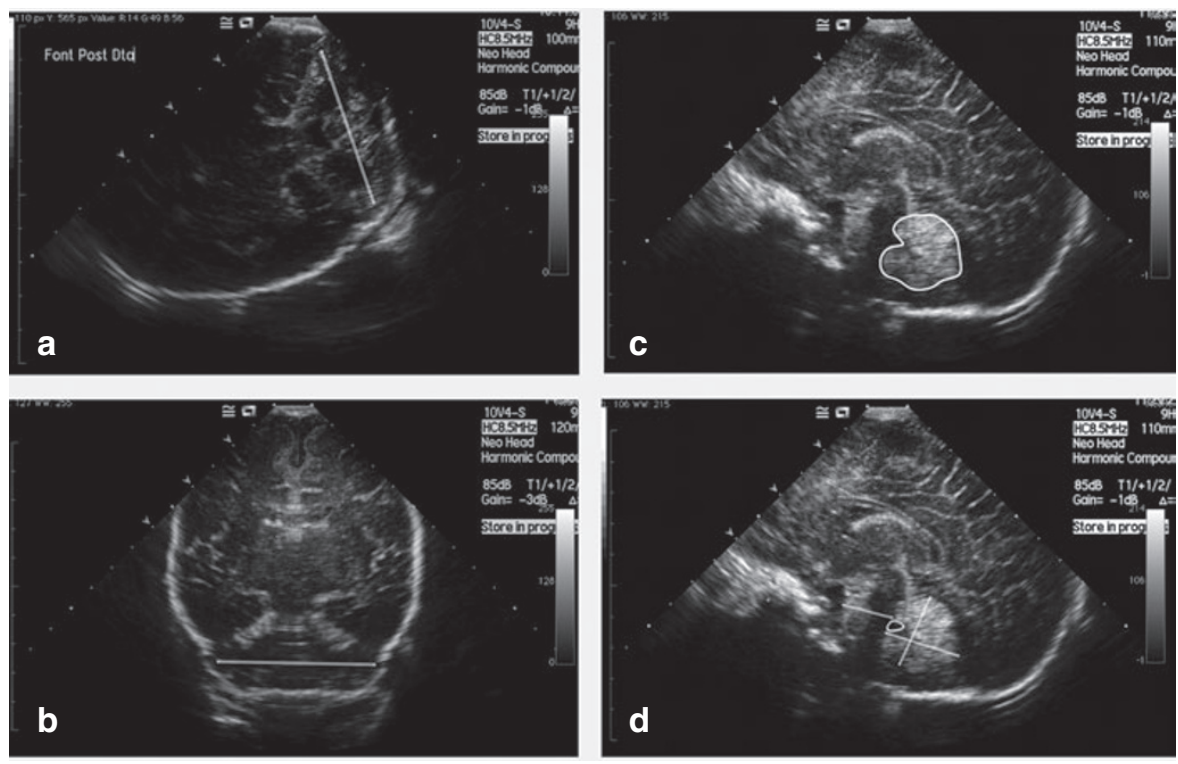

Figure 2. Examples of cerebellar images and the main measurements: (a) the coronal plane via the MF showing the TCD; (b) the coronal plane via the anterior fontanelle showing TCD; (c) the midsagittal plane via the anterior fontanelle showing CVA; (d) the midsagittal plane via the anterior fontanelle showing the $\mathrm{VH}$, fourth ventricle area, and APPD. APPD, anteroposterior pons diameter; APVD, anteroposterior vermis diameter; CVA, cerebellar vermis area; $\mathrm{TCD}$, transverse cerebellar diameter; $\mathrm{VH}$, vermis height.

\section{Measurements}

Measurements were performed by one author (A.M.G.) at a workstation using ImageArena software (TomTec Imaging Systems, Unterschleissheim, Germany) of the following structures and spaces (Figure 2):

1. TCD was measured from a coronal plane through the AF, according to the original description by Makhoul et al. (28) and/or from an axial plane through the MF, according to the original description by Davies et al. (29); we did not take both TCD measurements for all infants, but for most we used the measurement made using the AF view. In 19 preterm and 16 term infants, we had both measures, and in 10 infants, only from a mastoid view. We have previously shown that measurements made from the AF and MF views correlate very well on scans performed during the first postnatal week in preterm infants (23). In this study, we re-tested the comparability of both measurements in a sample of term- and preterm-born infants at TEA.

2. Measurements made in a midsagittal plane are as follows:

- Anteroposterior vermis diameter was measured according to the description by Cuddihy et al. (30)

- Vermis height was calculated according to the description by Pogliani et al. (31)

- CVA was automatically measured by the software after manual outlining of the vermis, excluding the cerebellar tonsils

- Anteroposterior pons diameter was measured linearly with calipers from the anterior border of the vermis above the fourth ventricle to the anterior limit of the pons, in a similar way to the measurement described for fetal ultrasound by Achiron et al. (32)

- Fourth ventricle area was automatically measured by the software after manual outlining of the fourth ventricle

- Fourth ventricle depth was measured linearly with calipers from the anterior base of the fourth ventricle to its posterior tip

- $\mathrm{CMH}$ was measured linearly with calipers from the intracranial border of the posterior lip of the foramen magnum to the lower border of the vermis; a neonatal measurement of the CMH was described in the 1980s, but the methodology used was built to deal with the technical problems of early scanners and cannot be reproduced using recent scanners. This measurement is not the same as that used fetally for measuring the cisterna magna.

Intracranial diameters were also measured from ultrasound images made via the AF in order to allow corrections of the cerebellar measurements for head shape, since it is recognized that preterm infants at TEA often have longer and narrower head shape as compared with term-born infants: (i) the biparietal diameter was measured from a coronal image at the level of the Sylvian fissures; (ii) the occipitalfrontal diameter was measured from a midsagittal image from the occipital bone to the frontal bone just above the orbits; and (iii) the intracranial height was measured from the posterior lip of the foramen magnum to the AF.

Statistical analysis was performed using IBM SPSS statistics version 19 (IBM, Armonk, NY). Sample distribution was assessed with Shapiro-Wilk test. Comparisons of numerical dependent variables between groups were performed either using independent samples $t$-test when both groups were normally distributed or using Mann-Whitney test when at least one of the groups did not follow a normal distribution. Corrections for confounding variables were done using ANCOVA models. Correlations between numerical explanatory variables and numerical dependent variables were performed using Pearson or Spearman correlations depending on the distribution of data. Multiple regression analysis was used to evaluate if significant explanatory variables on bivariate analysis remained significant when all explanatory variables were considered in a model.

The intra- and interobserver reliability of the cUS measurements was tested on repeated measurements (blinded to the original ones) using ICC mixed model for absolute agreement on at least $10 \%$ for intraobserver and $25 \%$ of the sample for interobserver agreement. The strength of agreement was classified using the scale described by Brennan and Silman (33). Accuracy was considered good for ICC between 0.61 and 0.80 and very good for ICC $>0.80$.

\section{ACKNOWLEDGMENTS}

The authors thank Ana Isabel Lopes and Jorge Campos for reviewing the manuscript.

\section{STATEMENT OF FINANCIAL SUPPORT}

No financial support was provided for this article. 


\section{REFERENCES}

1. Volpe J. The encephalopathy of prematurity-brain injury and impaired brain development inextricably intertwined. Semin Pediatr Neurol 2009;16(4):167-78.

2. Keunen K, Kersbergen KJ, Groenendaal F, Isgum I, de Vries LS, Benders MJ. Brain tissue volumes in preterm infants: prematurity, perinatal risk factors and neurodevelopmental outcome: a systematic review. J Matern Fetal Neonatal Med 2012;25:Suppl 1:89-100.

3. Haldipur P, Bharti U, Alberti C, et al. Preterm delivery disrupts the developmental program of the cerebellum. PLoS ONE 2011;6:e23449.

4. Schlapbach LJ, Adams M, Proietti E, et al.; Swiss Neonatal Network \& Follow-up Group. Outcome at two years of age in a Swiss national cohort of extremely preterm infants born between 2000 and 2008. BMC Pediatr 2012;12:198.

5. Moore T, Hennessy EM, Myles J et al. Neurological and developmental outcome in extremely preterm children born in England in 1995 and 2006: the EPICure studies. BMJ 2012;345:e7961.

6. de Vries LS, van Haastert IC, Benders MJ, Groenendaal F. Myth: cerebral palsy cannot be predicted by neonatal brain imaging. Semin Fetal Neonatal Med 2011;16:279-87.

7. Boardman JP, Craven C, Valappil S, et al. A common neonatal image phenotype predicts adverse neurodevelopmental outcome in children born preterm. Neuroimage 2010;52:409-14.

8. Mathur AM, Neil JJ, Inder TE. Understanding brain injury and neurodevelopmental disabilities in the preterm infant: the evolving role of advanced magnetic resonance imaging. Semin Perinatol 2010;34:57-66.

9. Inder TE, Warfield SK, Wang H, Hüppi PS, Volpe JJ. Abnormal cerebral structure is present at term in premature infants. Pediatrics 2005;115:28694.

10. Mewes AU, Hüppi PS, Als $\mathrm{H}$, et al. Regional brain development in serial magnetic resonance imaging of low-risk preterm infants. Pediatrics 2006;118:23-33.

11. Graça AM, Cardoso K, Costa J, Cowan FM. Cerebral volume at term age: Comparison between preterm and term-born infants using cranial ultrasound. Early Hum Dev 2013;89(9):643-8.

12. Horsch S, Muentjes C, Franz A, Roll C. Ultrasound diagnosis of brain atrophy is related to neurodevelopmental outcome in preterm infants. Acta Paediatr 2005;94:1815-21.

13. Lind A, Parkkola R, Lehtonen L, et al.; PIPARI Study Group. Associations between regional brain volumes at term-equivalent age and development at 2 years of age in preterm children. Pediatr Radiol 2011;41:953-61.

14. Srinivasan L, Allsop J, Counsell SJ, Boardman JP, Edwards AD, Rutherford M. Smaller cerebellar volumes in very preterm infants at term-equivalent age are associated with the presence of supratentorial lesions. AJNR Am J Neuroradiol 2006;27:573-9.

15. Shah DK, Anderson PJ, Carlin JB, et al. Reduction in cerebellar volumes in preterm infants: relationship to white matter injury and neurodevelopment at two years of age. Pediatr Res 2006;60:97-102.

16. Limperopoulos C, Soul JS, Gauvreau K, et al. Late gestation cerebellar growth is rapid and impeded by premature birth. Pediatrics $2005 ; 115$ : 688-95.
17. Limperopoulos C, Soul JS, Haidar H, et al. Impaired trophic interactions between the cerebellum and the cerebrum among preterm infants. Pediatrics 2005;116:844-50.

18. Limperopoulos C, Bassan H, Gauvreau K, et al. Does cerebellar injury in premature infants contribute to the high prevalence of long-term cognitive, learning, and behavioral disability in survivors? Pediatrics 2007;120:58493.

19. Allin M, Matsumoto H, Santhouse AM, et al. Cognitive and motor function and the size of the cerebellum in adolescents born very pre-term. Brain 2001;124(Pt 1):60-6.

20. Parker J, Mitchell A, Kalpakidou A, et al. Cerebellar growth and behavioural \& neuropsychological outcome in preterm adolescents. Brain 2008;131(Pt 5):1344-51.

21. Messerschmidt A, Prayer D, Brugger PC, et al. Preterm birth and disruptive cerebellar development: assessment of perinatal risk factors. Eur J Paediatr Neurol 2008;12:455-60.

22. Tam EW, Chau V, Ferriero DM, et al. Preterm cerebellar growth impairment after postnatal exposure to glucocorticoids. Sci Transl Med 2011;3:105ra105.

23. Graça AM, Cardoso K, Costa J, Cowan F. Assessment of gestational age using cerebellar measurements at cranial ultrasound: what is the best approach? Early Hum Dev 2013;89(1):1-5.

24. Van Kooij BJM, Benders MJNL, Anbeek P, Van Haastert IC, De Vries LS, Groenendaal F. Cerebellar volume and proton magnetic resonance spectroscopy at term, and neurodevelopment at 2 years of age in preterm infants. Dev Med Child Neurol. 2012;54(3):260-6.

25. Spittle AJ, Doyle LW, Anderson PJ, et al. Reduced cerebellar diameter in very preterm infants with abnormal general movements. Early Hum Dev 2010;86:1-5.

26. Fenton T. A new growth chart for preterm babies: Babson and Benda's chart updated with recent data and a new format. BMC Pediatr 2003;10:1-10.

27. The International Neonatal Network. The CRIB (clinical risk index for babies) score: a tool for assessing initial neonatal risk and comparing performance of neonatal intensive care units. Lancet 1993;342(8865):193-8.

28. Makhoul IR, Goldstein I, Epelman M, Tamir A, Reece EA, Sujov P. Neonatal transverse cerebellar diameter in normal and growth-restricted infants. J Matern Fetal Med 2000;9:155-60.

29. Davies MW, Swaminathan M, Betheras FR. Measurement of the transverse cerebellar diameter in preterm neonates and its use in assessment of gestational age. Australas Radiol 2001;45:309-12.

30. Cuddihy SL, Anderson NG, Wells JE, Darlow BA. Cerebellar vermis diameter at cranial sonography for assessing gestational age in low-birth-weight infants. Pediatr Radiol 1999;29:589-94.

31. Zuccotti GV, Pogliani L, Dilillo D, Lista G, Radaelli G. Nomogram of the cerebellar vermis height at birth in small-for-gestational-age neonates. Acta Paediatr 2008;97:745-50.

32. Achiron R, Kivilevitch Z, Lipitz S, Gamzu R, Almog B, Zalel Y. Development of the human fetal pons: in utero ultrasonographic study. Ultrasound Obstet Gynecol 2004;24:506-10.

33. Brennan P, Silman A. Statistical methods for assessing observer variability in clinical measures. BMJ 1992;304:1491-4. 